Rev. Bras. Saúde Prod. Anim., Salvador, v.14, n.3, p.558-564 jul./set., 2013 http://www.rbspa.ufba.br ISSN 15199940

\title{
Comparação entre protocolos de sincronização de cio para receptoras de embriões bovinos
}

\author{
Comparison of estrous synchronization protocols to bovine embryos recipients
}

NOGUEIRA, Ériklis ${ }^{1 *}$; PANTOJA, Thiago Augusto Rodrigues ${ }^{2}$; PEDROSO, Merlison Figueiredo $^{3}$; MARQUES JUNIOR, Heitor Romero ${ }^{2}$; BORGES, Juliana Corrêa ${ }^{4}$; DIAS, Alexandre Menezes ${ }^{5}$; ITAVO, Luis Carlos Vinhas ${ }^{6}$

\footnotetext{
${ }^{1}$ Embrapa Pantanal, Corumbá, Mato Grosso do Sul, Brasil.

${ }^{2}$ Universidade Católica Dom Bosco, Curso de Medicina Veterinária, Campo Grande, Mato Grosso do Sul, Brasil.

${ }^{3}$ Embriza, Tecnologia em Embriões, Campo Grande, Mato Grosso do Sul, Brasil.

${ }^{4}$ Universidade de São Paulo, Faculdade de Agronomia, Departamento de Produção Animal, Piracicaba, São Paulo, Brasil

${ }^{5}$ Universidade Federal de Mato Grosso do Sul, Faculdade de Medicina Veterinária e Zootecnia, Campo Grande, Programa de Pós-Graduação em Ciência Animal, Mato Grosso do Sul, Brasil.

${ }^{6}$ Universidade Católica Dom Bosco, Curso de Zootecnia, Campo Grande, Mato Grosso do Sul, Brasil.

*Endereço para correspondência: eriklis@cpap.embrapa.br
}

\section{RESUMO}

O trabalho foi conduzido com objetivo de avaliar dois protocolos de sincronização de cio em receptoras de embriões sobre a taxa de aproveitamento ao longo do ano, bem como os custos e as características reprodutivas dos animais sincronizados. Os tratamentos foram: T1: sincronização para transferência em tempo fixo com utilização de implantes de progesterona e estradiol, e T2: sincronização com aplicação de prostaglandina e observação de cio. Foram sincronizadas 2947 receptoras de embriões, e a taxa de aproveitamento foi de $83,24 \%$ no tratamento 1 , e $60,73 \%$ no tratamento 2. Os corpos lúteos foram avaliados no dia da inovulação de embriões, obtendo-se $63,73 \%$ da ovulação no ovário direito. As taxas de aproveitamento não apresentaram diferenças entre os meses do ano $(\mathrm{P}>0,05)$. O protocolo em tempo fixo apresentou custo mais elevado (US\$ 10,51 vs 2,03) que a utilização de prostaglandinas e observação de cio, no entanto a taxa de aproveitamento de receptoras foi maior. As taxas de aproveitamento das receptoras não variam ao longo do ano, quando atendidas as condições de nutrição e manejo adequados para receptoras de embrião bovino.

Palavras-chave: corpo lúteo, prostaglandina, receptoras, transferência de embriões

\section{SUMMARY}

The study was conducted to evaluate two protocols for synchronization of estrus in embryo recipients on the utilization rate throughout the year, as well as costs and the reproductive characteristics of synchronized animals. The treatments were: syncronization to transfer fixed-time with the use of implants of progesterone and oestradiol, and synchronization with prostaglandin and heat detection. 2947 embryo recipients were synchronized, and the rate of utilization was $83.24 \%$ in treatments 1 and $60.73 \%$ in treatments 2 . The corpus luteum were evaluated on the day of embryo transfer, yielding $63.73 \%$ of ovulation in the right ovary. The utilization rate did not differ among months. The protocol at fixed time showed higher cost (U.S. \$ 10.51 vs 2.03) than the use of prostaglandins and heat detection, but the utilization rate was higher . The utilization rates of the recipients do not vary throughout the year, when observing the conditions of nutrition and appropriate management for bovine embryo recipients.

Keywords: corpus luteum, embryo transfer, prostaglandins, recipients 


\section{INTRODUÇ̃̃̃O}

Atualmente, a transferência de embriões (TE) é uma das alternativas aplicadas no melhoramento bovino que possibilita retirar o máximo de descendentes de um animal de alto valor genético (MAPLETOFT, 2002).

Em um programa de TE, em muitos aspectos o manejo das receptoras é mais crítico para o sucesso do programa que a doadora, uma vez que é a receptora que deverá estabelecer e manter a gestação a termo, parir sem assistência e criar um bezerro de alto mérito genético (JONES \& LAMB, 2008). Assim a obtenção de receptoras aptas, os elevados custos para manutenção dessas receptoras aliados à baixa eficiência da detecção de cio (com ou sem aplicação de Prostaglandina $(\mathrm{PGF} 2 \alpha)$ ), podem limitar a aplicação e o sucesso desta biotecnologia (BO et al., 2002).

A sincronização de cio e da ovulação em um grupo de fêmeas permite que se estime o momento do cio com razoável precisão. $\mathrm{O}$ estabelecimento de protocolos de sincronização de ovulação e transferência de embriões em tempo fixo (TETF) tem colaborado desta maneira para aumentar as taxas de aproveitamento dos animais tratados (BARUSELLI et al., 2000). Como o resultado de prenhez de animais submetidos à sincronização de cio com aplicação de prostaglandina, ou com utilização de implantes de progesterona (P4) têm-se mostrado equivalentes em vários trabalhos (CHASE et al., 2009; BARREIROS et al., 2006), a taxa de aproveitamento é fator preponderante para o sucesso e custo da técnica. Neste sentido, Baruselli et al. (2004) observaram que 40 a $50 \%$ das receptoras tratadas com prostaglandina associada a detecção de cio, foram aproveitados para inovulação de embriões. Considerando $50 \%$ de taxa de concepção, espera-se uma taxa de prenhez final em torno de $25 \%$, o que onera os trabalhos de transferência de embriões, pelo alto custo de aquisição e manutenção destas receptoras.

Esta pesquisa foi conduzida com objetivo de comparar dois protocolos de sincronização de cio, sendo o primeiro, sincronização para transferência em tempo fixo com utilização de implantes de progesterona e estradiol, e o segundo sincronização com aplicação de prostaglandina e observação de cio, avaliando o aproveitamento ao longo do ano das receptoras de embrião, bem como as características reprodutivas dos animais sincronizados.

\section{MATERIAL E MÉTODOS}

Foram avaliadas 2947 receptoras de embriões entre vacas e novilhas em idade reprodutiva, no período de janeiro a outubro de 2008, em propriedades no estado do Mato Grosso do Sul. Foram selecionados os animais que apresentavam boa condição corporal (mínimo de 5 em escala de 1-9), classificação de consistência uterina tônica e corpo lúteo $(\mathrm{CL})$ característico ou folículos $<8 \mathrm{~mm}$, indicando ciclicidade. Os animais que apresentaram anormalidades detectáveis nos órgãos genitais foram descartados. As fêmeas foram mantidas em pastagens cultivadas dos gêneros Brachiaria sp. e Panicum sp. em taxa de lotação adequada para obtenção de desempenho produtivo satisfatório, recebendo suplementação mineral ou protéico-energética ad libitum e rigoroso controle sanitário. As receptoras utilizadas eram todas cruzadas (zebu $\mathrm{x}$ europeu) e foram 
submetidas a dois protocolos de sincronização:

Protocolo 1-T1 $(\mathrm{n}=$ 2728): Para a sincronização, os animais receberam dispositivo intravaginal novo ou usado, contendo $1 \mathrm{~g}$ de P4 (PRIMER ${ }^{\circledR}$, Tecnopec, São Paulo, Brasil) e 2mg de benzoato de estradiol (BE) (ESTROGIN®, Farmavet, São Paulo, Brasil), por via intramuscular (IM), em dia aleatório do ciclo estral (dia 0 ) após prévia avaliação ginecológica. No dia sete foi administrado $0,150 \mathrm{mg}$ de $d$-cloprostenol (prostaglandina- $\mathrm{PGF}_{2 \alpha}$ ) (PROLISE $\Re$, Tecnopec,São Paulo, Brasil) e 10mg de FSH (Folltropin ${ }^{\circledR}$,Bioniche Animal Health, Toronto, Canadá) por via IM. O dispositivo intravaginal foi removido no nono dia pela manhã e foi administrado $0,3 \mathrm{~mL}$ $(0,6 \mathrm{mg})$ de Cipionato de Estradiol

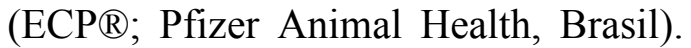
$\mathrm{O}$ cio sincronizado foi estimado para $\mathrm{o}$ dia onze.

Protocolo 2- T2 (n= 219): administração de $2 \mathrm{~mL}$ de Prostaglandina (PGF2 $\alpha$ PROLISE $®$, Tecnopec, São Paulo, Brasil) exógena nos animais que apresentarem corpo lúteo após a palpação retal, seguido de observação de cio em um período de 2 a 5 dias.

Nos dois protocolos, após oito dias da identificação das fêmeas em cio (protocolo 2), ou do cio estimado (protocolo 1), as fêmeas foram avaliadas por ultrassonografia (ultrasom Aloka, com probe linear de $5 \mathrm{mHZ}$ ), verificando-se a presença dos corpos lúteos para então ser realizada a transferência de embriões nos animais aptos.

A resposta ao tratamento foi realizada pela avaliação da relação corpo lúteo/ ovário, da seguinte forma: 1: CL maior do que o ovário, 2: $\quad \mathrm{CL}$ aproximadamente do mesmo tamanho que o ovário e 3: CL menor que o ovário, avaliando-se ainda o lado da ovulação ( $\mathrm{D}=$ direito, $\mathrm{E}=$ esquerdo)

Os corpos lúteos que apresentaram cavidades (corpos lúteos cavitários) durante o exame ultrassonográfico foram incluídos na contagem da respectiva classificação referente ao lado da ovulação e relação tamanho CL/ovário.

Alguns animais no processo de sincronização apresentaram mais de um corpo lúteo funcional, sendo classificados separadamente como superovulados (SOV) e, portanto, não entraram na contagem da relação $\mathrm{CL} /$ ovário. $\mathrm{O}$ custo dos tratamentos foi obtido pela avaliação dos preços em dólares (US\$) US\$ dos medicamentos utilizados nos protocolos.

Utilizou-se um delineamento em blocos ao acaso, e a análise estatística foi realizada aplicando-se o teste do Quiquadrado, com correção de Yates, com nível de significância de $5 \%(\mathrm{P}<0,05)$, utilizando-se o pacote estatístico SAS version 9.1. (SAS Institute, 2002).

\section{RESULTADOS E DISCUSSÃO}

O protocolo de sincronização em tempo fixo apresentou índice superior de aproveitamento de receptoras aptas para transferência, comparado ao protocolo utilizando prostaglandina e observação de cio $(\mathrm{P}<0,05)$, conforme mostra a Tabela 1.

Quadros et al. (2006), avaliaram a eficiência dos protocolos de sincronização de receptoras e obtiveram $76 \%$ de aproveitamento trabalhando com protocolo de tempo fixo associando progesterona e ECP, valores próximos aos encontrados neste experimento. Também Barreiros et al. (2006) obtiveram resultados semelhantes na avaliação ultrassonográfica para estimar a 
taxa de aproveitamento de receptoras tratadas com PGF2 $\alpha$ e P4, resultando em $72,8 \%$ e $79 \%$ de aproveitamento, respectivamente.

A grande variação na resposta de apresentação de cio com aplicação de PGF2 $\alpha$, deve-se, dentre outros fatores ao dia do ciclo estral em que se encontra a fềmea, no dia da aplicação do hormônio. Conforme Gioso et al. (2005), que observaram que a aplicação de luteolítico em diferentes fases de diestro interferem na taxa de sincronização.

Tabela 1.Número de receptoras sincronizadas e taxa de aproveitamento nos protocolos com uso de prostaglandina e sincronização em tempo fixo

\begin{tabular}{lccccc}
\hline Protocolo & $\begin{array}{c}\text { Animais } \\
\text { tratados }\end{array}$ & $\begin{array}{c}\text { Animais } \\
\text { aproveitados }\end{array}$ & $\%$ & $\begin{array}{c}\text { Animais } \\
\text { descartados }\end{array}$ & $\%$ \\
\hline Prostaglandina & 219 & 133 & $60,73^{\mathrm{b}}$ & 86 & $39,26^{\mathrm{b}}$ \\
Tempo fixo & 2728 & 2271 & $83,24^{\mathrm{a}}$ & 457 & $16,75^{\mathrm{a}}$ \\
Total & 2947 & 2404 & - & 543 & - \\
\hline
\end{tabular}

Letras diferentes nas colunas diferem estatisticamente $(\mathrm{P}<0,05)$.

Quando os animais pertencentes às classes luteais 2 e 3 foram agrupados (correspondendo, aos dias 11 a 16 do ciclo estral) e comparados aos da classe 1 (dias 07 a 10), as taxas de sincronização foram diferentes $(76,1 \%$ versus $55,5 \%$; $\mathrm{P}<0,05$ ), indicando que corpos lúteos em fases medianas e tardias respondem melhor à sincronização que animais em fase luteal inicial.

Em relação aos custos dos protocolos de sincronização, observou-se que o protocolo com administração de prostaglandina e observação de cio apresentou um custo médio de medicamentos de US\$ 2,03 por animal, no entanto faz-se necessário alto número de receptoras, pois nem todas terão corpo lúteo funcional para responder ao protocolo, adiciona-se a isto o custo da mão de obra para a detecção do cio.

O protocolo em tempo fixo apresentou custo médio de medicamentos de US\$ 10,51 por animal, possuindo benefícios como: reduzir o número de receptoras descartadas antes mesmo de entrar para o protocolo, por não apresentarem corpo lúteo, uma vez que propicia sincronização em qualquer fase do ciclo estral; elimina os erros de detecção de cio; aumenta a taxa de aproveitamento; e reduz a taxa de descarte, representando, portanto, um custo/benefício favorável para a propriedade.

As receptoras de embriões foram avaliadas mensalmente de acordo com o protocolo de sincronização utilizado. As taxas de aproveitamento nos diferentes meses não apresentaram diferença estatística $(\mathrm{P}>0,05)$ em ambos os tratamentos, apesar de índices de aproveitamento numericamente inferiores nos meses de julho e agosto (Tabela 2).

Rodrigues et al. (2007), avaliando a taxa de concepção na inseminação artificial e transferência de embriões de vacas holandesas de alta produção, verificaram que as taxas de concepção oscilaram ao longo dos meses do ano, e que as taxas de transferência de embriões foram menores nos meses de junho, julho e agosto demonstrando a influência climática nos resultados, o que não foi observado em nosso trabalho. 
Rev. Bras. Saúde Prod. Anim., Salvador, v.14, n.3, p.558-564 jul./set., 2013 http://www.rbspa.ufba.br ISSN 15199940

Tabela 2. Número de receptoras de embriões sincronizadas, aproveitadas e descartadas nos diversos meses do ano

\begin{tabular}{cccc}
\hline Mês & Receptoras sincronizadas & $\begin{array}{c}\text { Aproveitadas } \\
(\%)\end{array}$ & $\begin{array}{c}\text { Receptoras Descartadas } \\
(\%)\end{array}$ \\
\hline Jan & 183 & $148(80,87)$ & $35(19,13)$ \\
Fev & 621 & $520(83,74)$ & $101(16,26)$ \\
Mar & 247 & $216(87,45)$ & $31(12,55)$ \\
Abr & 379 & $320(84,43)$ & $59(15,57)$ \\
Mai & 199 & $162(81,41)$ & $37(18,59)$ \\
Jun & 236 & $205(86,86)$ & $31(13,14)$ \\
Jul & 515 & $375(72,82)$ & $140(27,18)$ \\
Ago & 155 & $117(75,48)$ & $38(24,52)$ \\
Set & 143 & $122(85,31)$ & $21(14,69)$ \\
Out & 269 & $219(81,41)$ & $50(18,59)$ \\
Total Geral & 2947 & $2404(81,57)$ & $543(18,43)$ \\
\hline Letras diferentes nas colunas diferem estatisticamente $(\mathrm{P}<0,05)$. &
\end{tabular}

Em relação às características, ginecológicas das receptoras, os corpos lúteos classificados como grau 2 foram encontrados em maior freqüência no ovário direito $(47,42 \%)$, apresentando também maior presença de corpos lúteos cavitários, onde $74,16 \%$ destes CLs foram encontrados no ovário D (Tabela 3). O total de CLs cavitários foi de $3,42 \%$. Barreiros et al. (2006), comparando as taxas de prenhez entre receptoras com corpos lúteos compactos e cavitários, observaram que os corpos lúteos cavitários apareceram em torno de $22 \%$ dos animais avaliados. Também Nogueira et al. (2012) encontram percentual de 27,6\% de CLs cavitários em receptoras de embriões, dados esses superiores aos encontrados nesta pesquisa. Segundos os autores acima este tipo de CL, não interfere negativamente na prenhez, apresentando ainda valores semelhantes nas concentrações plasmáticas de Progesterona nos animais avaliados.

Tabela 3. Lado de ovulação (ovário direito [D], ovário esquerdo [E]) e relação tamanho do corpo lúteo/ovário $(1,2,3)$, de receptoras que responderam ao tratamento de sincronização de cio

\begin{tabular}{lcc}
\hline Tipo de Ovulação e Relação CL/ ovário & Número de Animais & \% de Ocorrência \\
\hline D1 & 271 & 11,27 \\
D2 & 1140 & 47,42 \\
D3 & 121 & 5,03 \\
\hline Total D & 1532 & $63,72^{\mathrm{a}}$ \\
\hline E1 & 165 & 6,86 \\
E2 & 595 & 24,75 \\
E3 & 83 & 3,46 \\
\hline Total E & 843 & $35,07^{\mathrm{b}}$ \\
\hline Ovários Superovulados & 29 & $1,21^{\mathrm{c}}$ \\
\hline Total & 2404 & $100 \%$ \\
\hline
\end{tabular}

Letras diferentes nas colunas diferem estatisticamente $(\mathrm{P}<0,05)$. 
A presença de mais de um corpo lúteo no mesmo animal, classificado como Superovulado (SOV), ocorreu em apenas $1,21 \%$ dos animais avaliados. A baixa proporção de ovários SOV deveuse provavelmente a baixa dosagem de FSH utilizado (10mg).

Em relação ao lado da ovulação, foi encontrado que $63,72 \%$ das receptoras apresentaram ovulação, avaliada pela presença de CL, no ovário direito, e $35,06 \%$ das ovulações ocorreram no ovário esquerdo, apresentando diferença estatística $(\mathrm{P}<0,05)$, conforme também observado por Leal et al. (2009), que encontraram $63,3 \%$ das ovulações ocorrendo no ovário direito.

Também Kozicki et al. (2005) avaliando o efeito da somatotrofina bovina e sua relação com recrutamento folicular, verificaram que durante o exame ultrassonográfico realizado nos animais, $62,5 \%$ das ovulações ocorreram no ovário direito, enquanto que somente $37,5 \%$ no ovário esquerdo, concordando com nossos resultados.

O protocolo de sincronização em tempo fixo apresenta índice de aproveitamento superior quando comparado ao protocolo com utilização de prostaglandina, além de eliminar a necessidade de observação de cio. As ovulações ocorreram predominantemente no ovário direito, que apresentou também maior número de corpos lúteos cavitários.

As taxas de aproveitamento das receptoras não variam ao longo do ano, quando atendidas as condições de nutrição e manejo adequados para receptoras de embrião bovino.

\section{AGRADECIMENTOS}

À Empresa Embriza Biotecnologia, de Campo Grande-MS, pela cessão dos dados de acompanhamento das receptoras.
BARREIROS, T.R.R.; BLASCHI, W.; BORSATO, E.A.; LUDWIG, H.E.; MEIRA DA SILVA, D.R.; SENEDA, M.M. Comparação das taxas de prenhez entre receptoras com corpos lúteos cavitários ou compactos após protocolo de sincronização com cloprostenol ou transferência de embriões em tempo fixo. Semina: Ciências Agrárias, v.27, p.657664, 2006.

BARUSELLI, P.S.; MARQUES, M.O.; CARVALHO, N.A.T.; VALENTIM, R.; BERBER, R.C.A.; CARVALHO FILHO, A.F.; MADUREIRA, E.H.; COSTA NETO W.P. Ovsynch protocol with fixed-time embryo transfer increasing pregnancy rates in bovine recipients. Acta Scientiae Veterinariae, v.1, n.28, p.205, 2000. Suppl.

BARUSELLI, P.S.; REIS, E.L.; MARQUES, M.O.; NASSER, L.F.; BO, G.A. The use of hormonal treatments to improve reproductive performance of anestrous beef cattle in tropical climates. Animal Reproduction Science, v.82-83, p.479-486, 2004.

BO, G.A.; BARUSELLI, P.S.; MORENO, D.; CUTAIA, L.; CACCIA, M.; TRIBULO, R. The control of follicular wave development for selfappointed embryo transfer programs in cattle. Theriogenology, v.57, p.53-72, 2002.

CHASE, C.C.; VARGAS, C.A.; HAMMOND, A.C.; OLSON, T.A.; GRIFFIN, J.L.; MURPHY, C.N.; TEWOLDE, A.; FIELDS, M.J. Embryo transfer in angus and brahman recipient cows: Effect of two methods of estrus synchronization on induced estrus and pregnancy. Revista científica, v.19, n.6, p.630-638, 2009. 
GIOSO, M.M.; COSTA, E.P.; FERNANDES, A.C.C.; TORRES, C.A.A.; CARVALHO, G.R. Perfil de Progesterona e Intervalo ao Estro de Receptoras Bovinas Sincronizadas com Doses Reduzidas de Cloprostenol.

Revista Brasileira de Zootecnia, v.34, n.4, p.1181-1187, 2005.

JONES, A.L.; LAMB, G.C. Nutrition, synchronization, and management of beef embryo transfer recipients.

Theriogenology, v.69, p.107-115, 2008.

KOZICKI, L.E.; SEGUI, M.S.; FANTINI FILHO, J.C2; PRADO, F.R.A.; MATTÉ, F.; GLASER JR, P.; WEISS, R.R. A somatotrofina bovina (bst) e sua relação com o recrutamento folicular ovariano durante o ciclo estral de vacas. Archives of Veterinary Science, v.10, n.1, p.35-44, 2005.

LEAL, L.S.; OBA, E.; FERNANDES, C.A.C.; SA FILHO, O.G. Avaliação do corpo lúteo, contratilidade uterina e concentrações plasmáticas de progesterona e estradiol em receptoras de embriões bovinos. Ciência Animal Brasileira, v.10, n.1, p.174-183, 2009.

MAPLETOFT, R.J.; STEWARD, K.B.; ADAMS, G.P. Recent advances in the superovulation of cattle. Reproduction Nutrition Development, v.42, p.1-11, 2002.
NOGUEIRA, E.; CARDOSO, G.S.; MARQUES JUNIOR, H.R.; DIAS, A.M., ÍTAVO, L.C.V.; BORGES, J.C. Effect of breed and corpus luteum on pregnancy rate of bovine embryo Recipients. Revista Brasileira de Zootecnia, v.41, n.9, p.2129-2133, 2012.

QUADROS, F.A.; MELO STERZA, F.A.; SANTOS, E.S.; FONSECA, A.; BAUDRAZ, J.A.; MARQUES, V.T.; FERRI, F.; MARQUES, M.O.; SILVA, R.C.P.; BARUSELLI, P.S. Avaliação da eficiência dos protocolos de sincronização de receptoras de embriões bovinos para TETF, utilizando Benzoato de Estradiol e Cipionato de Estraciol. Acta Scientiae Veterinariae, v.34, p.498, 2006. Supl. 1

RODRIGUES, C.A.; AYRES, H.; FERREIRA, R.M.; TEIXEIRA, A.A.; MANCILHA, R.F.; OLIVEIRA, M.E.F.; SOUZA, A.H.; BARUSELLI, P.S. Taxa de concepção à inseminação artificial e à transferência de embriões em vacas holandesas de alta produção.

Acta Scientiae Veterinariae, v.35, p.1254, 2007. Supl. 3

SAS Institute. Statistical analysis system: user's guide: statistics. Version 9.1. Cary, 2002.

Data de recebimento: 14/05/2013

Data de aprovação: 25/09/2013 